\title{
POLÍTICA EDUCATIVA EN PERSPECTIVA HISTÓRICA. TEXTOS ESCOGIDOS
}

Por Manuel de Puelles Benítez. Madrid: Biblioteca Nueva (colección Memoria y crítica de la educación, 28), 2017. 392 páginas. ISBN 978-84-16938-53-7.

Editar un libro que recoge una selección de textos publicados con anterioridad es una empresa arriesgada que los coordinadores de este proyecto han gestionado con acierto al asegurarse que los temas presentados abordan problemas cruciales de la política educativa de los dos últimos siglos sumamente interrelacionados y algunos pendientes aún de solución. Además, como el autor ha supervisado personalmente la selección de los trabajos que integran este volumen, la vertebración temática de los asuntos tratados en él está plenamente garantizada. Todos ellos se centran en cuestiones que atienden a un mismo orden y están estructurados en torno a los procesos de construcción y desarrollo de los sistemas educativos contemporáneos, por lo que la presencia de dos elementos tan íntimamente relacionados como la educación y la ideología aflora desde la primera de sus páginas, impregnando la totalidad de su contenido.

Asumiendo que el sistema educativo español no surge de un modo aislado, aunque su evolución presenta especificidades genuinas, Manuel de Puelles presenta en primer lugar un marco de referencia para el lector cuyas coordenadas están determinadas por la penetración en España de la modernidad que alumbró la Revolución Francesa, una modernidad política que hace referencia al Estado liberal y a la influencia de los principios de libertad e igualdad aplicados a la educación. El autor nos sitúa frente a los grandes principios revolucionarios - libertad, igualdad, nación- y su relación con la educación, cerrando el círculo con un epígrafe centrado en la Revolución francesa y el sistema educativo español donde la libertad de enseñanza y la religión se perfilan como protagonistas de los grandes debates sobre los principios fundamentales de la política de la educación, controversias que marcarán el rumbo de la evolución de 
nuestro sistema educativo y permanecerán vigentes hasta nuestros días sin que, a pesar de haber transcurrido más de doscientos años, hayamos sido capaces de tejer un consenso sobre el que cimentar un pacto que permita dar una respuesta satisfactoria a esta confrontación.

Con esta estrategia Manuel de Puelles persigue dos objetivos. Por un lado, señala el lugar exacto donde anclar un extremo del hilo argumental cuyo ovillo devanará a lo largo de las páginas del libro, indicando al lector el norte que guiará sus pasos cuando aborde los diferentes trabajos que lo integran. Además, indica dónde volver la vista en caso de pérdida, ya que este marco histórico de la modernidad política es el crisol donde se funde el contenido de unos textos cuya principal característica es su coherencia interna desde una perspectiva histórica. Por otro lado, este marco teórico proporciona al lector las claves necesarias para captar el alcance y la permanencia en el tiempo de la reacción frente a la modernidad de dos de los más conspicuos, activos y recalcitrantes conspiradores contra su avance: la Iglesia católica y las fuerzas políticas conservadoras.

La hoja de ruta que traza está diseñada para que el lector recale en momentos trascendentales de la evolución de nuestro sistema educativo, y se detenga en cambios especialmente significativos acaecidos en diferentes etapas de nuestra Historia y, para lograrlo, se centra en cuatro cuestiones fundamentales.

En primer lugar, ahonda en la relación histórica que existe entre Estado y educación, afirmando que repensar el papel del Estado y de la escuela pública constituye actualmente una exigencia no sólo urgente sino también inaplazable. Seguidamente lleva a cabo un análisis de la problemática del profesorado de educación secundaria y bachillerato, concluyendo que la cultura escolar de los profesores de secundaria sigue anclada mayoritariamente en una concepción del bachillerato como nivel propedéutico, actitud que entra en conflicto con el concepto de una educación secundaria obligatoria como prolongación de la primaria que, hoy por hoy, parece irreversible. Sumado a ello, la formación del profesorado de secundaria sigue siendo especializada, académica y centrada fundamentalmente en contenidos curriculares. El tercer asunto que aborda Manuel de Puelles en este bloque es el debate sobre la cuestión de los recursos en la España del primer tercio del siglo xx, un asunto crucial directamente relacionado con la debilidad del sistema educativo 
en su conjunto, especialmente para la etapa de la educación primaria. Finalmente, el cuarto y último punto está dedicado a la reforma educativa de 1970 que restauró el concepto de la educación como servicio público.

Fijado el marco teórico, y analizados varios de los momentos más cruciales de la evolución de nuestro sistema educativo, el autor presenta, estructurados en dos bloques perfectamente delimitados (políticas de libertad e igualdad y políticas de secularización y laicidad), un conjunto de trabajos que aportan al lector las claves necesarias para acercarse a los grandes temas de la política actual de la educación. Manuel de Puelles lleva a cabo un análisis pormenorizado de alguno de los debates históricamente más trascendentales y pertinaces para la evolución de nuestro sistema educativo, todos ellos plenamente vigentes en nuestros días. Así, por ejemplo, ante los ojos del lector se desgranan, desde una perspectiva histórica, cuestiones tan determinantes para «la salud» de nuestro sistema educativo como las relaciones entre educación, igualdad y libertad, la revolución social que supuso la escolaridad obligatoria y su influencia en el currículo, la escuela comprensiva como instrumento para proporcionar a la ciudadanía una formación general no sólo extensa sino también intensa, la diversidad como una realidad inherente a la sociedad y a la escuela que es consustancial a la comprensividad desde su propio nacimiento, y las políticas neoliberales que impregnan desde la década de los ochenta del pasado siglo la mayoría de los sistemas educativos europeos apostando por la libertad de elección de centro, el cheque escolar y la privatización de la educación, con un objetivo claro: restar competencias al Estado.

De entre todos estos debates destaca por su persistencia, permanencia e intensidad el que se ocupa del lugar que debe tener la religión en la escuela, las relaciones Iglesia-Estado en materia escolar, y las tensiones generadas por el proceso de secularización registrado en momentos históricos tan significativos como el periodo de cambios del siglo XIX al Xx, donde confluye el proceso de fundación de un nuevo Estado y el de formación de un nuevo sistema de educación pública, la Segunda República Española en la que convergieron distintas tendencias y soplaron por vez primera en nuestra historia auténticos aires de renovación para nuestro sistema educativo, la dictadura franquista o la denominada Transición democrática. 
El broche final del libro gira en torno a la posibilidad y la necesidad de alcanzar un consenso en materia de educación, así como de los límites que deben caracterizar al mismo. Bajo el título de "Consenso y política educativa», Manuel de Puelles presenta dos trabajos cuyo contenido converge en la necesidad de alcanzar un nuevo pacto escolar que permita conformar un sistema educativo de calidad cuyos beneficios alcancen a toda la ciudadanía.

En el primero de ellos — «El pacto educativo: del consenso constitucional al disenso político»—- pone de relieve la sobrecarga ideológica preconstitucional que durante la Transición a la democracia estuvo presente en todos los ámbitos de la vida española, un lastre que todas las fuerzas políticas tuvieron que lanzar por la borda para poder alcanzar un difícil consenso que desembocó en un pacto escolar de alto contenido ideológico que condicionó indudablemente tanto el proceso como el producto final. Lo cierto es, como subraya el autor, que el artículo 27 de la Constitución de 1978 además de un pacto escolar fue un pacto abierto a la alternancia política, una verdad incontrovertible que ha acarreado una enorme carga de dificultades para su desarrollo político y legislativo.

Además de pasar revista a las características del consenso constitucional y a las dificultades para alcanzar un acuerdo básico en educación, Manuel de Puelles analiza también el alcance y los límites de un consenso básico constitucional que no impide el disenso de las políticas prácticas, planteando interrogantes sobre la dificultad de conciliar políticas públicas de educación y ahondando en la complejidad del binomio consenso-disenso. Como en democracia ningún grupo ejerce el poder permanentemente, sino que el ejercicio del mismo es resultado del voto popular, la alternancia democrática derivada de esta circunstancia puede dificultar la reforma de la educación por lo que el autor pone de relieve la necesidad imperiosa de alcanzar un pacto de Estado en educación cimentado en un nuevo consenso social, político y económico.

En el segundo trabajo — «Reflexiones sobre la necesidad de un nuevo pacto escolar: del disenso al consenso»-, subraya que el artículo 27 de la Constitución de 1978 fue obra de un consenso tejido en torno a dos valores esenciales, la libertad y la igualdad, aunque la materialización de este consenso básico fue cada vez más problemática, desvirtuándose progre- 
sivamente por la aplicación de múltiples leyes y la aparición de diversas políticas educativas aplicadas a lo largo de estos cuarenta años, lo cual no es óbice para no haya gozado de virtualidad en la práctica política.

Ante la cuestión de si es posible o no alcanzar un pacto en educación, Manuel de Puelles afirma que, a pesar de existir una fuerte demanda social en este sentido, es necesario alcanzar un consenso constitucional en educación que refuerce las garantías de los derechos básicos que se derivan de los principios de libertad de enseñanza y de igualdad ante la educación. Sin embargo, aclara también que no se trata de formular un nuevo consenso ideológico como el que representó el artículo 27, que en lo sustancial se debe mantener, sino de alcanzar un pacto de mínimos sobre políticas que mejoren la educación y que, en consecuencia, puedan ser asumidas por los partidos políticos en el marco de la alternancia democrática.

Para el autor, este acuerdo debe contemplar tres vertientes y programarse en una serie de pasos bien definidos. En primer lugar, un acuerdo previo sobre el diagnóstico serio y riguroso de la educación en España. Tras el diagnóstico, un acuerdo sobre los puntos débiles del sistema que hay que mejorar, y, en tercer y último lugar, un acuerdo sobre las políticas de reforma encaminadas a fortalecer el sistema educativo y a resolver los problemas detectados. Como resultado de este proceso, los principios derivados de este triple acuerdo podrían incorporarse a la reforma constitucional del artículo 27, reforzando así el marco garantista de los derechos de libertad y de igualdad establecidos en él.

En definitiva, con esta selección de textos, Manuel de Puelles mueve al lector a una reflexión sobre cuestiones vitales de política educativa que han protagonizado un debate que sigue activo tras doscientos años de historia, analizando detenidamente su impacto en la génesis de nuestro sistema educativo. Además, aporta también muchas de las claves necesarias para trabajar en pos de un consenso que permita alcanzar un pacto educativo cimentado sobre el derecho a la educación y la libertad de enseñanza, un pacto que permita a la ciudadanía y a la clase política que la representa asimilar definitivamente que, a pesar de la omnipotente presencia de la ideología en la educación, es posible, a través del diálogo y el consenso, que ésta deje de vivirse definitivamente como un problema. 
El planteamiento de Manuel de Puelles se asemeja a la estrategia de Ariadna para ayudar al joven Teseo a escapar del laberinto después de acabar con el Minotauro. En nuestro caso, el monstruo toma carta de naturaleza en la falta de un acuerdo de mínimos capaz de preparar y facilitar una reforma constitucional, un desencuentro al que se han sacrificado once leyes de educación, tres de ellas universitarias, desde el año 1978. El extremo del ovillo que permitirá salir del laberinto está firmemente anclado en los derechos de libertad e igualdad recogidos en el artículo 27 de la Constitución y enunciados, hace ya doscientos años, por los revolucionarios franceses que alumbraron la modernidad política.

Es importante también prestar atención a otra de las razones que los coordinadores aportan al lector sobre el propósito perseguido con la publicación de este libro. Debido a su dispersión, muchos de los textos seleccionados para formar parte de esta edición son difíciles de localizar y algunos están incluidos en revistas que no se ocupan propiamente del estudio de temas educativos. De ahí precisamente la importancia y el acierto de haber reunido en este volumen unos trabajos destacados, pero poco accesibles, en los que Manuel de Puelles aborda desde una perspectiva histórica una serie de cuestiones educativas cuyo origen se remonta doscientos años atrás, pero que, sin embargo, han llegado hasta nosotros plenamente vigentes porque la virtualidad de las relaciones entre ideología, política y educación permanece durante amplios períodos de tiempo al tratarse de fenómenos de extensa duración.

Otro acierto de los coordinadores ha sido incluir al final del volumen una selección de obras del autor, lo que facilita al lector la localización y consulta de las mismas, así como la posibilidad de poder ampliar el estudio de alguna de las cuestiones tratadas en Política educativa en perspectiva histórica. Textos escogidos.

En mi opinión, la selección de trabajos que integran este volumen, todos ellos relacionados con la Política y la Historia de la Educación y organizados en torno a los procesos de construcción y desarrollo de los sistemas educativos contemporáneos, aportan muchas de las claves que ayudarán al lector a entender la génesis de nuestro sistema público de educación, una cuestión que, a pesar de encontrarnos ya en pleno siglo XXI, no ha sido esclarecida aún satisfactoriamente debido, en parte, a la complejidad de los factores políticos, económicos, sociales y culturales 
que intervienen de forma interrelacionada en la comprensión de este fenómeno histórico.

Aunque este libro fue concebido como un homenaje que sus compañeros del Departamento de Historia de la Educación y Educación Comparada de la UNED y del Centro de Investigación MANES quisieron brindarle con motivo de su jubilación —sólo administrativa porque Manuel de Puelles sigue desarrollando una fecunda actividad investigadora y colaborando activamente con distintos organismos como el Colectivo Lorenzo Luzuriaga-, lo cierto es que su contenido trasciende este objetivo y su lectura ayudará a estudiantes y estudiosos de la educación española a reflexionar desde una perspectiva histórica sobre los problemas educativos del presente, a entender mejor las peculiaridades de nuestro sistema educativo, y a afrontar con garantías el reto de un futuro mejor para la educación de nuestro país.

José Ramón López Bausela Inspector de Educación jr.porrero@gmail.com 